\title{
4q25 and ZFHX3 Single Nucleotide Polymorphisms are Associated with Electroanatomical Characteristics of Left Atrium and Clinical Outcomes of Radiofrequency Catheter Ablation in Patients with Atrial Fibrillation
}

Jaemin Shim, MD1; Jae-Sun Uhm, $\mathrm{MD}^{2}$; Boyoung Joung, MD, PhD2; Moon-Hyoung Lee, MD, PhD2; HuiNam Pak, MD, PhD²

1Division of Cardiology, Korea University Anam Hospital; 2Division of Cardiology, Yonsei University College of Medicine, Seoul, Republic of Korea
Received: June 23, 2016

Revision Received: September 6, 2016

Accepted: September 20, 2016

Correspondence: Hui-Nam Pak, MD, PhD.

50, Yonsei-ro, Seodaemun-gu, Seoul 03722,

Republic of Korea

Tel: +82-2-2228-8459 Fax: +82-2-393-2041

E-mail: hnpak@yuhs.ac

Copyright (C) 2016 The Official Journal of Korean Heart Rhythm Society Editorial Board and MMK Communications Limited

\section{ABSTRACT}

Background and Objectives: Previous studies have demonstrated an association between several single nucleotide polymorphisms and atrial fibrillation (AF). We hypothesized that the phenotypes of AF patients were associated with common AF susceptibility alleles.

Subjects and Methods: A total of 659 patients $(57 \pm 9$ years, $76 \%$ male) with AF who underwent catheter ablation and 659 age, and sex-matched controls were genotyped for the common AF susceptibility alleles rs2200733 and rs6843082 at 4q25, rs2106261 at ZFHX3, and rs13376333 at KCNN3. The phenotypes of AF patients, including electroanatomical characteristics of the left atrium and recurrence after ablation, were compared.

Results: The rs2200733 variant allele carriers have larger left atrium volume $(128.5 \pm 40.7$ vs. $113.5 \pm 29.2 \mathrm{~mL}, p=0.020)$, longer PR interval $(185.9 \pm 32.6$ vs. $174.8 \pm 21.9 \mathrm{~ms}, p=0.018)$, and higher amplitude of negative P-wave terminal force in Lead $V_{1}(0.07 \pm 0.04$ vs. $0.05 \pm 0.04 \mathrm{mV}, p=0.015)$ on electrocardiography than those without the variant allele. When the patients were assigned to three groups according to the number of variant alleles (Group A: no variant, $\mathrm{n}=15$; Group B: 1 variant, $\mathrm{n}=158$; Group C: 2 variants, $\mathrm{n}=439$ ), incremental prognostic value, according to the number of variant alleles, was demonstrated (Log Rank $p=0.015$ ). Multivariate Cox regression analysis showed that persistent $\mathrm{AF}(\mathrm{OR} 1.677,95 \% \mathrm{Cl}$ $1.176-2.381, p=0.004)$ and the number of variants (OR 1.552, 95\% $\mathrm{Cl} 1.099-2.222, p=0.015)$ were independent predictors for recurrence of $\mathrm{AF}$.

Conclusion: This study showed the common AF susceptibility alleles at $4 \mathrm{q} 25$ and $Z F H X 3$ are associated with electroanatomical characteristics of the left atrium and the clinical outcomes of catheter ablation in Korean patients with AF.

Key Words: - Atrial Fibrillation - Single Nucleotide Polymorphisms - Catheter Ablation 


\section{Introduction}

Atrial fibrillation (AF) is the most common form of cardiac arrhythmia in clinical practice and causes significant morbidity and mortality. ${ }^{1}$ Although many risk factors for AF have been identified, including advanced age, hypertension, structural heart disease, and congestive heart failure, a subset of younger subjects develop AF in the absence of established risk factors. The mechanisms underlying AF are not fully understood, but growing evidence supports an underlying heritable component and that genetic variation may play a role in the pathogenesis of $\mathrm{AF}^{2}$ Familial cases among young patients with lone AF underscore a genetic basis for the disease, and research implicates pathogenic mutations and risk-conferring functional polymorphisms in AF development. Recently, genome-wide association studies (GWAS) performed in populations of European-descent have identified several genetic variants associated with AF. ${ }^{3}$ To date, three genomic regions / candidate genes have shown strong association with AF: 4q25, near paired-like homeodomain transcription factor 2 (PITX2); zinc finger homeobox 3 (ZFHX3) on 16q22; and potassium intermediate/small conductance calcium-activated channel, subfamily $\mathrm{N}$, member 3 (KCNN3) on $1 \mathrm{q} 21.4,5$ The $4 \mathrm{q} 25$ genetic locus is adjacent to PITX2, a transcription factor that determines left-right asymmetry of the heart. ${ }^{6}$ Recently, non-coding single nucleotide polymorphisms (SNPs) on chromosome 4q25 have been associated with the occurrence of AF in different populations. ${ }^{4,7}$ The sequence variant $r s 2106261$ is an intronic SNP located in the ZFHX3 gene, also called AT motif-binding factor 1 (ATBF1), on chromosome $16 \mathrm{q} 22$, and was highly associated with AF in a Chinese GeneID study. ${ }^{8}$ The SNP in KCNN3, on chromosome 1q21, was most significantly associated with lone AF in people of European ancestry in a study of 1,335 individuals with lone AF and 12,844 unrelated individuals without AF.' One of the longterm risk factors for $\mathrm{AF}$ is prolonged $\mathrm{PR}$ interval on electrocardiography (ECG)..$^{10}$ Increased P-wave duration is indicative of abnormal phenotypic characteristics such as left or right atrial hypertrophy and or enlargement, which reflect delayed intra- or inter-atrial conduction. ${ }^{11}$ Multiple reports have also shown the value of $\mathrm{P}$-wave prolongation as a predictor of $\mathrm{AF}$ after cardiac interventions such as coronary artery bypass surgery. ${ }^{12}$
Genetic variants at $4 \mathrm{q} 25$ and $16 \mathrm{q} 22$, and PR intervals, have also been reported to predispose individuals to AF. ${ }^{13}$ Recently, AF has been most effectively treated with radiofrequency catheter ablation (RFCA), a procedure that isolates and terminates the source of ectopic activity. ${ }^{14}$ The use of RFCA is increasing worldwide. Given the cost and potential complications of this procedure, analysis of pre-procedural characteristics that stratify the procedural efficacy and safety might be helpful. Interestingly, the $4 \mathrm{q} 25$ SNP has been recently reported to predict the recurrence of AF after RFCA. ${ }^{15}$ Here, we hypothesized that the phenotypes of AF patients, including electroanatomical characteristics of the left atrium (LA) and recurrence after RFCA, were modulated by the common AF susceptibility alleles, rs2200733 and rs6843082 at 4q25, rs2106261 at ZFHX3, and rs 13376333 at KCNN3.

\section{Materials and Methods}

\section{Study Population}

The study protocol was approved by the Institutional Review Board of the Yonsei University Health System and adhered to the Declaration of Helsinki. All patients provided written informed consent. Participants included 659 Korean patients with AF who underwent RFCA and 659 age and sex-matched healthy controls who were included in the Ansan-Ansung cohort study in the Korean National Institute of Health (KNIH) database. Exclusion criteria were: 1) permanent AF refractory to electrical cardioversion; 2) LA anteroposterior diameter $>55 \mathrm{~mm}$ as measured by echocardiogram; 3) AF with rheumatic valvular disease; 4) associated significant structural heart disease; and 5) prior AF ablation or maze surgery. Three-dimensional (3D) spiral computed tomography (CT) scans (64 Channel, Light Speed Volume CT, Philips, Brilliance 63, the Netherlands) were performed to visually define the pulmonary vein (PV) and LA anatomy. Trans-thoracic echocardiography (Sonos 5500, Philips Medical System, Andover, MA, USA or Vivid 7, GE Vingmed Ultrasound, Horten, Norway) was performed to evaluate the presence of structural heart disease, degree of LA remodeling, or ventricular function. Using trans-esophageal echocardiography, we ensured that no LA thrombus was present. All antiarrhythmic 
drugs were discontinued for at least five half-lives. Amiodarone was discontinued at least four weeks prior to the procedure. Anticoagulation therapy was maintained before RFCA.

\section{Electrophysiologic Mapping and Radiofrequency Catheter Ablation}

Intracardiac electrograms were recorded using the Prucka CardioLab $^{\mathrm{TM}}$ electrophysiology system (General Electric Health Care System Inc., Milwaukee, WI, USA). Catheter ablation was performed in all patients using 3D electroanatomical mapping (St. Jude Medical Inc., Minnetonka, MN, USA) merged with 3D spiral CT. If the initial recording identified a sinus rhythm, an LA $3 \mathrm{D}$ electroanatomical voltage map was generated during atrial pacing by obtaining contact bipolar electrograms from 350-400 points on the LA endocardium. The bipolar electrograms were filtered from $32-300 \mathrm{~Hz}$. For patients with the initial rhythm of AF during the procedure, we generated voltage maps during pacing following internal electrical cardioversion (2 to $10 \mathrm{~J}$, biphasic shocks with $\mathrm{R}$ wave synchronization, anodal decapolar catheter in high right atrium to cathodal duo-decapolar catheter inside of the coronary sinus, Lifepak12, Physiocontrol Ltd.). We did not obtain an LA voltage map if the frequently re-initiating AF required electrical cardioversion more than three times. We generally performed RFCA in sinus rhythm after electrical cardioversion. However, if sinus rhythm could not be maintained due to immediate recurrence of AF, RFCA was performed while in AF. We used an open irrigated-tip catheter (Celsius, Johnson \& Johnson Inc.; Diamond Bar, CA, USA; irrigation flow rate 20 to $30 \mathrm{~mL} / \mathrm{min} ; 30 \mathrm{~W} ; 47^{\circ} \mathrm{C}$ ) to deliver radiofrequency energy for ablation (Stockert generator, Biosense Webster Inc.; Diamond Bar, CA, USA). All patients initially underwent circumferential $\mathrm{PV}$ isolation and bi-directional block of the cavo-tricuspid isthmus. For patients with paroxysmal AF, additional linear ablation or complex fractionated atrial electrogram (CFAE) ablation, guided by the 3D-CFAE-cycle length map, was conducted at the operator's discretion. In patients with persistent AF, circumferential PV isolation, cavo-tricuspid isthmus block, roof line, posterior inferior line, and anterior line were performed as a standard lesion set. If AF persisted, CFAE ablation was done depending on the operator's decision. If AF persisted after internal cardioversion, despite administration of the aforementioned ablation protocols, procedures were stopped. The procedural endpoint was defined as when there was no immediate recurrence of AF after cardioversion during isoproterenol infusion (5-20 $\mu \mathrm{g} /$ $\mathrm{min})$. If non-PV foci were identified under isoproterenol, they were ablated.

\section{Post-ablation Follow-up}

Patients were asked to visit an outpatient clinic one, three, six, and 12 months after RFCA and every six months thereafter for follow-up. ECG was performed at each visit and anytime the patient reported palpitations. A Holter ECG (24- or 48-hour) and/or event recorder was performed on patients at every sixmonth follow-up, and whenever the patient complained of symptoms, for at least two years in accordance with the 2012 ACC/AHA/ESC expert consensus guidelines for AF management. ${ }^{14}$ We defined recurrence of $\mathrm{AF}$ as any episode of $\mathrm{AF}$ or atrial tachycardia of at least 30 seconds in duration. If $\mathrm{AF}$ was found on ECG during follow-up within three-months of RFCA, the patient was diagnosed with early recurrence of AF. Any AF recurrence thereafter was diagnosed as clinical recurrence and antiarrhythmic medications were prescribed.

\section{Off-line Analyses of Color-coded 3D Maps and CT Im- ages}

Color-coded voltage maps were generated by recording bipolar electrograms and measuring peak-to-peak voltage during high right atrial pacing with a cycle length of $500 \mathrm{~ms}$. We analyzed the color-coded voltage maps, that had been converted to image files, of both anterior-posterior (AP) and posterior-anterior (PA) views. PVs were not included in the analysis. Digital measurements of color-coded voltage maps were performed by a single investigator blinded to the clinical information of the maps, using a consistent method. The percentage of color-coded areas in each quadrant of the voltage maps was analyzed using customized software (Image Pro), and referenced to the color scale bars. Low voltage areas were coded gray and defined as LA voltage $\leq 0.2 \mathrm{mV}$. High voltage areas were coded purple and defined as LA voltage $>5.0 \mathrm{mV}$. The mean LA voltage was calculated by adding the area of each color (as a 
percentage), multiplying by the corresponding voltage, and dividing by the total area of LA. The reference distance was measured by the inter-electrode distances of coronary sinus catheters (duodecapolar catheter, St. Jude Medical Inc. Minnetonka, MN, USA.). To calculate local conduction velocity, the conduction distance was measured on the AP and PA views of the isochronal map, and the measured distance divided by the time difference. The 3D spiral CT images of LA were analyzed on an image processing workstation (Aquarius, Terarecon Inc., USA). Each LA image was divided into portions according to embryological origin as follows: the venous LA (posterior LA including the antrum and posterior wall), anterior LA (excluding the LA appendage and venous LA), and LA appendage. The absolute and relative volumes of each portion were calculated and compared.

\section{Electrocardiography}

Standard 12 lead ECGs were recorded for a few hours to a day before the catheter ablation procedure. Digitally stored ECGs were magnified on a high resolution computer screen and measured with a computerized caliper (Cardio Calipers, ver 3.3, ICONICO, NY, USA). Lead I, II, III, aVL, aVF, and Lead V were measured using Cardio Calipers. PR intervals were automatically measured with the GE Marquette MAC 5000 (GE Marquette Inc., Milwaukee, WI). P wave durations in Lead I, II,
III, aVL, and aVF were measured from the onset of the $\mathrm{P}$ wave to the return to the baseline. $P$ wave amplitudes were measured from the crest of the $P$ wave to the trough of the wave. The biphasic deflection in Lead $V_{1}$ was determined from the following: the duration of the initial positive deflection as measured from the onset of the $\mathrm{P}$ wave to the return to its baseline, before the onset of the negative deflection; the duration of negative $P$ wave deflection as calculated by subtracting the duration of the positive deflection from the duration of the entire $\mathrm{P}$ wave; and the amplitude of the terminal negative deflection as measured from the trough of the wave to the baseline (Figure 1).

\section{Genotyping}

Whole blood samples were collected using EDTA as an anticoagulant and DNA was extracted for genetic analyses. Genotyping of 4q25 rs2200733, PITX2 rs6843082, ZFHX3 rs2106261, and KCNN3 rs13376333 was performed using validated TaqMan assays (Life Technologies, Carlsbad, CA, USA). PCR reaction mixtures contained $0.9 \mu \mathrm{m}$ each of the forward and reverse primers, $0.2 \mu \mathrm{m}$ each of the fluorescein amidite and VIC minor groove binder sequence-specific probes, 3 ng DNA, $5.0 \mathrm{mM} \mathrm{MgCl}_{2}$, and 1xTaqMan Universal PCR Master Mix containing AmpliTaq gold DNA polymerase in a $5.5 \mu \mathrm{L}$ reaction volume. All SNPs had a call rate of $>99 \%$. All genotyping was performed by laboratory personnel blinded to all clinical data.
A PR interval=138 ms (CC genotype)

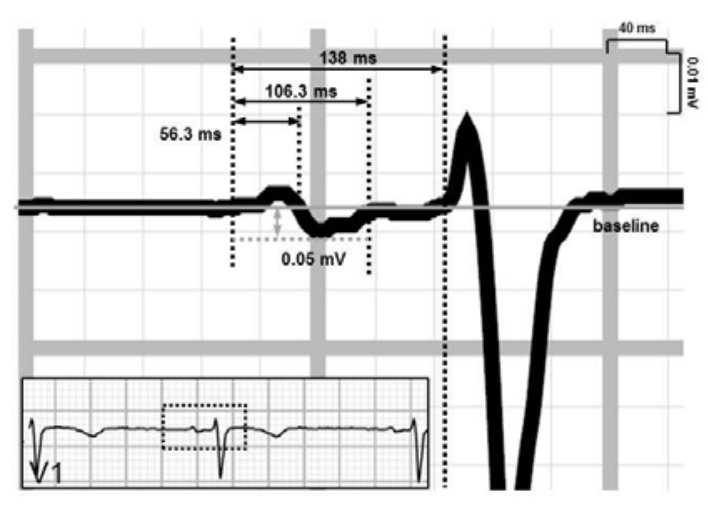

B PR interval=218 ms (TT genotype)

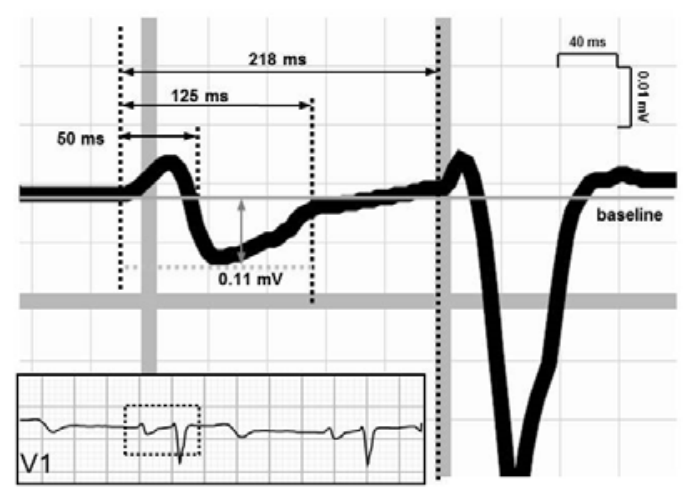

Figure 1. Measurement of $P R$ interval, $P$ wave duration, and $P$ wave amplitude in Lead $V_{1}$. Representative images of a patient with rs2200733 CC genotype (A) and TT genotype (B) are shown. 


\section{Statistical analysis}

All values are expressed as the mean \pm standard deviation. Multiple variables including clinical features, echocardiography parameters, and degree of electroanatomical remodeling of LA were compared according to different genotypes. The Student's t-test, Mann-Whitney U test, or Kruskal-Wallis $\mathrm{H}$ test was used, as appropriate, for comparisons between continuous data. The chisquare test or Fisher's exact test were used for comparisons between the categorical data. A chi-square test was used to test if the genotype distribution deviated from Hardy-Weinberg equilibrium. Kaplan-Meier survival analysis with a log rank test was used to present the recurrence of AF. Multivariate analysis was performed with the Cox stepwise forward regression model to determine the independent predictors of the recurrence of $\mathrm{AF}$ and odds ratios (ORs) using 95\% confidence intervals (CIs). P-values less than 0.05 was considered statistically significant. All statistical analyses were performed using SPSS version 20.0 (SPSS Inc. Chicago, IL, USA).

\section{Results}

\section{Clinical characteristics}

All AF patients underwent RFCA, 457 of them had paroxysmal atrial fibrillation (PAF) and 202 had persistent AF (PeAF). The baseline characteristics of the AF patients and controls are summarized in Table 1.

Frequency of genotypes in Korean patients with AF vs. controls

Table 2 summarizes the frequency of the genotypes of the common AF susceptibility alleles. 4q25 rs2200733 TT genotype ( 51.5 versus $25.8 \%, p<0.001$ ), CC genotype ( 7.7 versus $23.4 \%$, $p<0.001)$, PITX2 rs6843082 GG genotype (81.0 versus $49.8 \%$, $p<0.001)$, AA genotype (1.4 versus $8.8 \%, p<0.001), Z F H X 3$ rs2106261 AA genotype (24.5 versus $9.1 \%, p<0.001)$, and GG genotype (26.3 versus $49.5 \%, p<0.001$ ) were significantly

Table 1. Baseline characteristics of AF patients and control

\begin{tabular}{lccc}
\hline & AF $(\mathrm{n}=659)$ & Control $(\mathrm{n}=659)$ & Pvalue \\
\hline Age (years) & $57 \pm 9$ & $57 \pm 9$ & 0.989 \\
Male gender (\%) & 75.8 & 75.8 & 1.000 \\
BMI (kg/m²) & $24.8 \pm 2.8$ & NA & NA \\
PAF (\%) & 68.3 & NA & NA \\
CHF $(\%)$ & 2.5 & NA & NA \\
Hypertension (\%) & 45.1 & NA & NA \\
Age $>75(\%)$ & 3.4 & NA & NA \\
DM (\%) & 13.0 & NA & NA \\
Stroke or TIA (\%) & 10.5 & NA & NA \\
CHADS 2 score & $0.85 \pm 1.02$ & NA & NA \\
LA size $(m m)$ & $41.5 \pm 6.3$ & NA & NA \\
LVEF $(\%)$ & $63.7 \pm 7.9$ & NA & NA \\
\hline
\end{tabular}

AF, atrial fibrillation; BMI, body mass index; CHF, congestive heart failure; DM, diabetes mellitus; LVEF, left ventricular ejection fraction; NA, not applicable; PAF, paroxysmal atrial fibrillation; TIA, transient ischemic attack. 
different between the patient and control group. In contrast, the distribution of rs13376333 genotype was not significantly different between patients and control.

Comparison of AF patients with and without the rs2200733 variant

There were no significant differences in clinical and electroanatomical parameters between the rs6843082 SNP variant and non-variant groups. The comparison of $\mathrm{AF}$ patients with the non-risk allele of rs2200733 (CC genotype) and risk allele (TT+CT genotype) is shown in Table 3. Patients with the risk allele ( $\mathrm{T}$ ) exhibited a lower rate of CHF (2.1 versus 8.0\%, $p=0.012$ ). rs2200732 variant allele carriers had larger LA volume than non-variant carriers $(128.5 \pm 40.7$ versus $113.5 \pm 29.2 \mathrm{~mL}$, $p=0.005)$. When considering ECG parameters, the risk allele was associated with longer PR interval (185.9 \pm 32.6 versus $174.8 \pm 2$ $1.9 \mathrm{~ms}, p=0.018)$, longer duration of negative $\mathrm{P}$ terminal force in $\mathrm{V}_{1}(57.3 \pm 24.3$ versus $48.8 \pm 23.9 \mathrm{~ms}, p=0.018)$, and higher amplitude of negative $P$ terminal force in $V_{1}(0.07 \pm 0.04$ versus $0.05 \pm 0.04 \mathrm{mV}, p=0.015)$. In addition, risk allele carriers tended to have a higher, but not statistically significant, incidence of AF recurrence.

Comparison of AF patients with and without the rs2106261 variant

The characteristics of AF patients, according to rs2106261 genotype, were compared (Table 4). The AA genotype carriers showed shorter P-wave duration in Lead aVL (93.5 \pm 22.5 versus

Table 2. Genotype distribution

\begin{tabular}{lccc}
\hline & AF $(n=659)$ & Control $(n=659)$ & Pvalue \\
\hline rs2200733 (4q25) & 51.5 & 25.8 & $<0.001$ \\
TT (\%) & 40.8 & 50.8 & $<0.001$ \\
CT (\%) & 7.7 & 23.4 & $<0.001$ \\
CC (\%) & & & \\
rS6843082 (PITX2) & 81.0 & 49.8 & $<0.001$ \\
GG (\%) & 17.6 & 41.4 & $<0.001$ \\
GA (\%) & 1.4 & 8.8 & $<0.001$ \\
AA (\%) & & & $<0.001$ \\
IS2106261 (ZFHX3) & & 49.5 & 0.004 \\
GG (\%) & 26.3 & 41.3 & $<0.001$ \\
AG (\%) & 49.2 & 9.2 & \\
AA (\%) & 24.5 & & 0.215 \\
IS13376333 (KCNN3) & & 96.2 & 0.165 \\
GG (\%) & 94.8 & 3.6 & 0.320 \\
GA (\%) & 5.2 & 0.2 & \\
AA (\%) & 0.0 & & \\
\hline
\end{tabular}

$A F$, atrial fibrillation. 
Table 3. Comparison of AF patients with and without the rs2200733 variant

\begin{tabular}{|c|c|c|c|}
\hline & $\begin{array}{c}4 \mathrm{q} 25 \text { rs2200733 CC } \\
(\mathrm{n}=50)\end{array}$ & $\begin{array}{c}4 \mathrm{q} 25 \text { rs } 2200733 \mathrm{TT}+\mathrm{CT} \\
(\mathrm{n}=609)\end{array}$ & $P$ value \\
\hline Age (years) & $57 \pm 12$ & $57 \pm 11$ & 0.831 \\
\hline Male gender (\%) & 70.0 & 76.3 & 0.320 \\
\hline PAF (\%) & 74.0 & 67.6 & 0.350 \\
\hline BMI (kg/m²) & $24.5 \pm 3.2$ & $24.8 \pm 2.7$ & 0.562 \\
\hline $\mathrm{CHADS}_{2}$ score & $1.1 \pm 1.2$ & $0.83 \pm 1.0$ & 0.051 \\
\hline $\operatorname{CHF}(\%)$ & 8.0 & 2.1 & 0.012 \\
\hline Hypertension (\%) & 56.0 & 44.4 & 0.112 \\
\hline Age $>75(\%)$ & 6.0 & 3.8 & 0.434 \\
\hline DM (\%) & 10.0 & 8.4 & 0.522 \\
\hline Stroke/TIA (\%) & 6.0 & 1.6 & 0.195 \\
\hline \multicolumn{4}{|l|}{ ECG parameters } \\
\hline $\mathrm{PR}(\mathrm{ms})$ & $174.8 \pm 21.9$ & $185.9 \pm 32.6$ & 0.018 \\
\hline P dur II (ms) & $121.5 \pm 20.9$ & $122.2 \pm 20.2$ & 0.795 \\
\hline$P \operatorname{dur} V_{1}(+)(m s)$ & $66.3 \pm 20.8$ & $58.5 \pm 21.0$ & 0.012 \\
\hline$P$ dur $V_{1}(-)(m s)$ & $48.8 \pm 23.9$ & $57.3 \pm 24.3$ & 0.018 \\
\hline P amp II (mV) & $0.13 \pm 0.06$ & $0.14 \pm 0.05$ & 0.202 \\
\hline P amp $V_{1}(-)(m V)$ & $0.05 \pm 0.04$ & $0.07 \pm 0.04$ & 0.015 \\
\hline \multicolumn{4}{|l|}{ Echo parameters } \\
\hline LA size (mm) & $41.64 \pm 6.45$ & $41.48 \pm 6.23$ & 0.866 \\
\hline $\operatorname{LVEF}(\%)$ & $62.94 \pm 9.44$ & $63.70 \pm 7.73$ & 0.509 \\
\hline $\mathrm{E} / \mathrm{E}^{\prime}$ & $10.68 \pm 4.59$ & $10.09 \pm 4.00$ & 0.339 \\
\hline \multicolumn{4}{|l|}{ LA volume on $\mathrm{CT}(\mathrm{mL})$} \\
\hline LA & $113.5 \pm 29.2$ & $128.5 \pm 40.7$ & 0.005 \\
\hline Venous LA & $35.9 \pm 12.4$ & $40.7 \pm 15.4$ & 0.053 \\
\hline LA appendage & $9.7 \pm 4.4$ & $10.4 \pm 5.2$ & 0.420 \\
\hline Anterior LA & $68.8 \pm 20.2$ & $76.0 \pm 28.1$ & 0.111 \\
\hline \multicolumn{4}{|l|}{ LA voltage (mV) } \\
\hline LA & $1.18 \pm 0.50$ & $1.19 \pm 0.62$ & 0.966 \\
\hline Venous LA & $1.00 \pm 0.68$ & $0.99 \pm 0.80$ & 0.954 \\
\hline LA appendage & $2.25 \pm 1.52$ & $2.36 \pm 1.41$ & 0.634 \\
\hline Anterior LA & $1.12 \pm 0.47$ & $1.03 \pm 0.57$ & 0.377 \\
\hline \multicolumn{4}{|c|}{ Conduction velocity (m/sec) } \\
\hline Anterior & $0.59 \pm 0.29$ & $0.56 \pm 0.29$ & 0.547 \\
\hline Posterior & $0.56 \pm 0.29$ & $0.61 \pm 0.38$ & 0.515 \\
\hline \multicolumn{4}{|l|}{ Procedure } \\
\hline Procedure time (min) & $181.4 \pm 29.6$ & $191.8 \pm 47.7$ & 0.131 \\
\hline
\end{tabular}




\begin{tabular}{lccc} 
Fluoroscopic time (min) & $45.8 \pm 15.9$ & $47.4 \pm 26.2$ & 0.681 \\
Ablation time (min) & $82.8 \pm 25.9$ & $87.2 \pm 26.8$ & 0.258 \\
Recurrence & & & \\
Early recurrence (\%) & 30 & 31 & 0.823 \\
Clinical recurrence (\%) & 16 & 28 & 0.067 \\
\hline
\end{tabular}

$A F$, atrial fibrillation; BMI, body mass index; CHF, congestive heart failure; CT, computed tomography; DM, diabetes mellitus; ECG, electrocardiogram; LA, left atrium; LVEF, left ventricular ejection fraction; PAF, paroxysmal atrial fibrillation; TIA, transient ischemic attack.

98.6 $\pm 19.3 \mathrm{mV}, p=0.026)$ and higher venous $\mathrm{LA}$ voltage $(1.18 \pm 0.97$ versus $0.94 \pm 0.71 \mathrm{mV}, p=0.023$ ) (Figure 2). In addition, clinical recurrence occurred more frequently in patients with the AG + GG genotype than the AA genotype (28 versus $20 \%, p=0.049$ ).

\section{Characteristics and predictors of clinical recurrence of AF after RFCA}

Clinical recurrence of AF after RFCA was observed in 26.2\% of patients. We compared the characteristics of AF patients with and without clinical recurrence of AF (Table 5). Patients with recurrence were more likely to have PeAF ( 46.3 versus $23.5 \%$, $p<0.001)$, larger LA ( $42.8 \pm 6.4$ versus $41.1 \pm 6.2 \mathrm{~mm}, p=0.002)$, and longer PR interval $(189.9 \pm 31.2$ versus $183.4 \pm 32.7 \mathrm{~ms}$, $p=0.027)$. Procedure time $(p<0.001)$, fluoroscopic time $(p=0.003)$, and ablation time $(p<0.001)$ were all significantly longer in patients with recurrence of AF. The ZFHX3 rs2106261 variant allele was more prevalent in patients with $\mathrm{AF}$ recurrence ( 81.5 versus $73.7 \%, p=0.049$ ). We performed Kaplan-Meier survival analysis according to genotypes (Figure 3 ). During an $18.8 \pm 8.5$ month follow-up period patients with the $4 \mathrm{q} 25$ rs2200733 risk allele $(\mathrm{T})$ showed a trend of higher risk of recurrence (Log Rank $p=0.061$, Figure 3A). ZFHX3 rs 2106261 variant allele carriers had significantly higher recurrence rate than non-variant ( $\log \operatorname{Rank} p=0.015$, Figure $3 \mathrm{~B})$. When patients were assigned to three groups according to the number of variant alleles (Group A: no variant, $\mathrm{n}=15$; Group B: 1 variant, $\mathrm{n}=158$; and Group C: 2 variants, $n=439)$ there were no significant differences in age, gender, paroxysmal AF, CHADS 2 (congestive heart failure, hypertension, age $=75$ years, diabetes mellitus, stroke) score, LA size, and medications. However, venous LA volume was significantly greater in patients with the variant allele (Group A versus B \& C; 16.9 versus 22.3 and $22.9 \mathrm{~mL} / \mathrm{m}^{2} ; p=0.023$ \& 0.020 , respectively). Kaplan-Meier survival analysis showed incremental prognostic value according to the number of variant alleles (Figure 4, Log Rank $p=0.015$ ). Univariate Cox regression analysis identified that PeAF, LA size, and the number of variants in rs2200733 and rs2106261 were associated with the recurrence of AF after RFCA. Multivariate analysis showed that PeAF (OR $1.677,95 \% \mathrm{CI} 1.176-2.381, p=0.004)$ and the number of variants (OR 1.552, 95\% CI 1.099-2.222, $p=0.015$ ) were independent predictors for $\mathrm{AF}$ recurrence (Table 6).

\section{Discussion}

Here, we found that polymorphisms 4q25 PITX2 and 16q22 $Z F H X 3$, detected from peripheral blood, were associated with AF. The 4q25 rs2200733 and ZFHX3 rs2106261 genotypes were associated with electroanatomical remodeling of LA. The presence of the variant allele of $Z F H X 3$ rs 2106261 was also associated with AF recurrence after RFCA.

\section{AF is a hereditable disease and related to genetic poly- morphisms}

AF is known to be a degenerative disease with a multi-factorial background, such as aging and oxidative stress. Growing evidence shows that genetic variation may also play a role in the pathogenesis of AF. ${ }^{2}$ Fox et al. ${ }^{16}$ reported that a parental history of AF increased the risk of AF 1.9 times. Meanwhile, Lubitz et al. ${ }^{17}$ showed a $39 \%$ increase in the incidence of AF in patients with a family history of AF in their $1^{\text {st }}$ degree relatives. Recently, genome- 
Table 4. Comparison of AF patients with and without the rs2106261 variant

\begin{tabular}{|c|c|c|c|}
\hline & $\begin{array}{c}\text { ZFHX3 rs2106261 AA } \\
(n=161)\end{array}$ & $\begin{array}{c}\text { ZFHX3 rs2106261 AG+GG } \\
(\mathrm{n}=498)\end{array}$ & $P$ value \\
\hline Age (years) & $57 \pm 12$ & $56 \pm 11$ & 0.174 \\
\hline Male gender (\%) & 74.5 & 76.1 & 0.579 \\
\hline $\operatorname{PAF}(\%)$ & 65.2 & 69.1 & 0.393 \\
\hline BMI (kg/m²) & $24.4 \pm 2.9$ & $24.8 \pm 2.8$ & 0.084 \\
\hline $\mathrm{CHADS}_{2}$ score & $0.93 \pm 1.05$ & $0.83 \pm 1.01$ & 0.257 \\
\hline CHF (\%) & 3.4 & 2.3 & 0.402 \\
\hline Hypertension (\%) & 50.0 & 43.6 & 0.145 \\
\hline Age $>75(\%)$ & 5.7 & 2.7 & 0.059 \\
\hline DM (\%) & 12.1 & 13.4 & 0.666 \\
\hline Stroke/TIA (\%) & 8.0 & 9.1 & 0.677 \\
\hline \multicolumn{4}{|l|}{ ECG parameters } \\
\hline $\mathrm{PR}(\mathrm{ms})$ & $185.0 \pm 32.2$ & $183.1 \pm 37.1$ & 0.542 \\
\hline P dur aVL (ms) & $93.5 \pm 22.5$ & $98.6 \pm 19.3$ & 0.026 \\
\hline$P$ dur $V_{1}(+)(m s)$ & $56.2 \pm 19.9$ & $59.9 \pm 21.3$ & 0.164 \\
\hline$P$ dur $V_{1}(-)(m s)$ & $57.0 \pm 23.0$ & $56.6 \pm 24.7$ & 0.690 \\
\hline P amp aVL (mV) & $0.07 \pm 0.03$ & $0.08 \pm 0.03$ & 0.631 \\
\hline P amp $V_{1}(-)(m V)$ & $0.07 \pm 0.04$ & $0.07 \pm 0.04$ & 0.432 \\
\hline \multicolumn{4}{|l|}{ Echo parameters } \\
\hline LA size (mm) & $41.1 \pm 6.0$ & $41.6 \pm 6.3$ & 0.313 \\
\hline $\operatorname{LVEF}(\%)$ & $63.5 \pm 7.8$ & $63.7 \pm 7.9$ & 0.752 \\
\hline $\mathrm{E} / \mathrm{E}^{\prime}$ & $10.6 \pm 4.6$ & $9.9 \pm 3.8$ & 0.077 \\
\hline \multicolumn{4}{|l|}{ LA volume on CT (mL) } \\
\hline LA & $126.3 \pm 41.4$ & $127.7 \pm 39.7$ & 0.721 \\
\hline Venous LA & $41.0 \pm 17.4$ & $40.3 \pm 14.6$ & 0.628 \\
\hline LA appendage & $9.7 \pm 5.2$ & $10.5 \pm 5.0$ & 0.113 \\
\hline Anterior LA & $73.8 \pm 27.1$ & $75.8 \pm 27.9$ & 0.488 \\
\hline \multicolumn{4}{|l|}{ LA voltage (mV) } \\
\hline LA & $1.28 \pm 0.67$ & $1.15 \pm 0.58$ & 0.059 \\
\hline Venous LA & $1.18 \pm 0.97$ & $0.94 \pm 0.71$ & 0.023 \\
\hline LA appendage & $2.49 \pm 1.43$ & $2.28 \pm 1.40$ & 0.170 \\
\hline Anterior LA & $1.11 \pm 0.60$ & $1.01 \pm 0.55$ & 0.087 \\
\hline \multicolumn{4}{|c|}{ Conduction velocity (m/sec) } \\
\hline Anterior & $0.54 \pm 0.33$ & $0.51 \pm 0.33$ & 0.367 \\
\hline Posterior & $0.58 \pm 0.42$ & $0.55 \pm 0.40$ & 0.529 \\
\hline
\end{tabular}


Procedure

Procedure time (min)

$193.8 \pm 49.3$

$190.3 \pm 45.8$

0.408

Fluoroscopic time (min)

$49.9 \pm 42.3$

$46.5 \pm 17.1$

0.147

Ablation time (min)

$87.3 \pm 27.1$

$86.8 \pm 26.7$

0.850

Recurrence

Early recurrence (\%)

32

31

0.844

Clinical recurrence $(\%)$

20

28

0.049

AF, atrial fibrillation; BMI, body mass index; CHF, congestive heart failure; CT, computed tomography; DM, diabetes mellitus; ECG, electrocardiogram; LA, left atrium; LVEF, left ventricular ejection fraction; PAF, paroxysmal atrial fibrillation; TIA, transient ischemic attack.

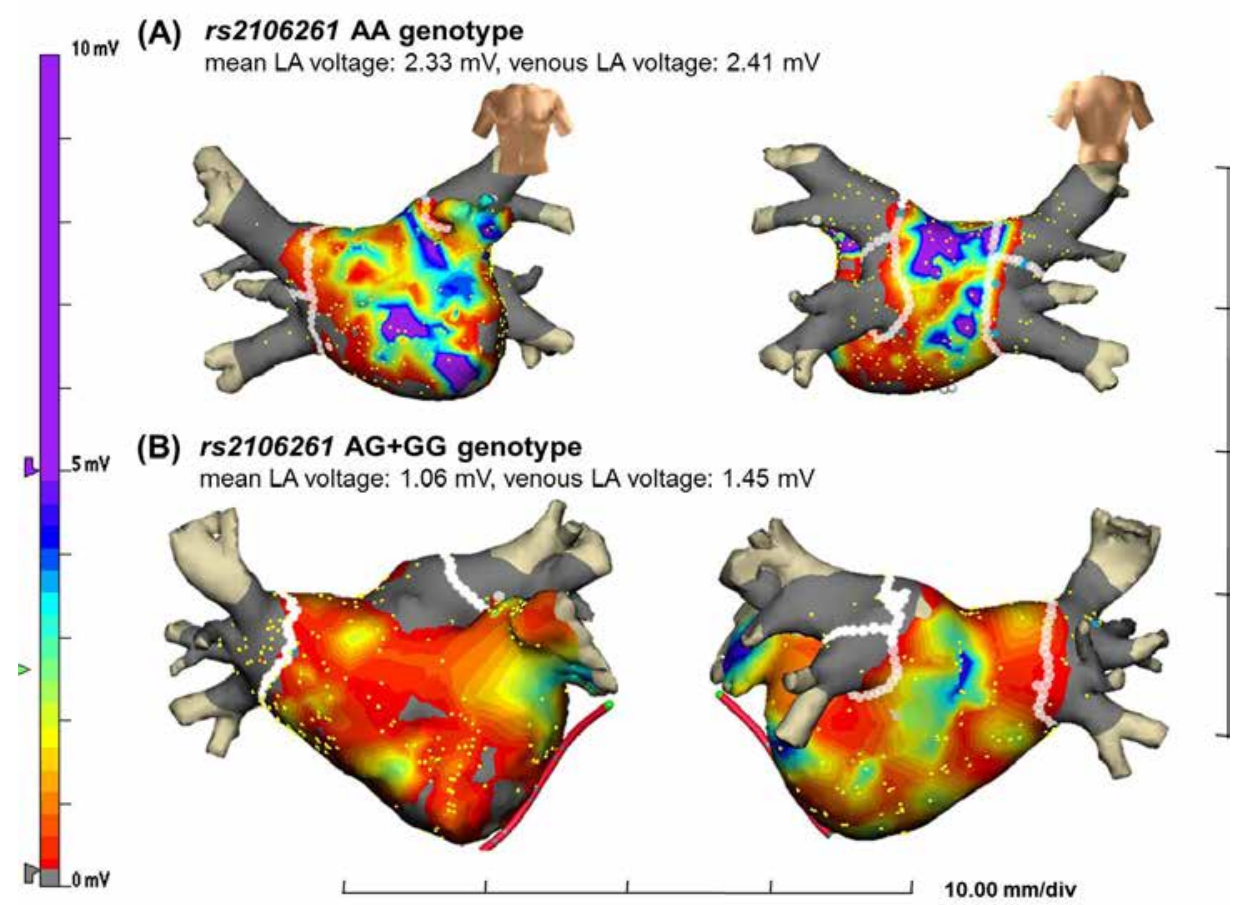

Figure 2. Representative images of color-coded $3 D$ voltage map of $L A$. $L A$ voltage, especially venous $L A$ voltage, is higher in a patient with rs2106261 AA genotype (A) than $A G+G G(B)$.

$L A$, left atrium

wide association studies have revealed several nuclear polymorphisms significantly associated with AF. These include polymorphisms in genes such as KCNN3 on chromosome 1q21, PITX2 on chromosome 4q25, and ZFHX3 on chromosome 16q22. ${ }^{4,5}$ Common SNPs in a non-coding region of chromosome $4 \mathrm{q} 25$ have been shown to be associated with AF, and rs2200733 is the most reliable genetic variant associated with AF. ${ }^{3}$ SNPs found in the 4q25 region are near PITX2, a gene believed to be involved in cardiac development, sinus node development, and susceptibility to atrial arrhythmias. ${ }^{18}$ There is emerging evidence that highly conserved non-coding regions may act as regulatory elements and contribute to phenotypic diversity. ${ }^{19}$ However, the mechanism by which genetic variation at the chromosome $4 \mathrm{q} 25$ locus leads to AF remains to be elucidated. Here, we evaluated the robust SNPs associated with AF: rs2200733 and rs6843082 at chromosome 4q25, rs2106261 at ZFHX3, and rs13376333 at KCNN3. The AF risk allele of SNPs rs2200733 and rs2106261 are much more frequent among Chinese subjects, in both affected and unaffected individuals $s^{7,8}$ in concordance with our results. Contrary to the results of other studies, in our study population, 
Table 5. Comparison of AF patients with and without clinical recurrence of AF

\begin{tabular}{|c|c|c|c|}
\hline & Recur $(+)(n=165)$ & $\operatorname{Recur}(-)(n=486)$ & $P$ value \\
\hline Age (years) & $58 \pm 11$ & $57 \pm 11$ & 0.312 \\
\hline Male gender (\%) & 78.0 & 74.8 & 0.402 \\
\hline $\operatorname{PAF}(\%)$ & 53.7 & 76.5 & $<0.001$ \\
\hline BMI (kg/m²) & $24.9 \pm 3.0$ & $24.8 \pm 2.6$ & 0.749 \\
\hline $\mathrm{CHADS}_{2}$ score & $0.87 \pm 0.99$ & $0.84 \pm 1.03$ & 0.714 \\
\hline CHF (\%) & 2.8 & 2.4 & 0.754 \\
\hline Hypertension (\%) & 45.2 & 45.7 & 0.918 \\
\hline Age $>75(\%)$ & 5.1 & 3.7 & 0.427 \\
\hline $\mathrm{DM}(\%)$ & 14.1 & 12.6 & 0.618 \\
\hline Stroke/TIA (\%) & 23.2 & 20.7 & 0.791 \\
\hline \multicolumn{4}{|l|}{ ECG parameters } \\
\hline $\mathrm{PR}(\mathrm{ms})$ & $189.9 \pm 31.2$ & $183.4 \pm 32.7$ & 0.027 \\
\hline P dur II (ms) & $124.2 \pm 21.4$ & $121.7 \pm 19.9$ & 0.173 \\
\hline$P$ dur $V_{1}(+)(m s)$ & $60.4 \pm 17.9$ & $58.6 \pm 22.2$ & 0.326 \\
\hline$P$ dur $V_{1}(-)(m s)$ & $57.1 \pm 25.2$ & $56.6 \pm 24.2$ & 0.813 \\
\hline$P$ dur $V_{1}(+/-)(m s)$ & $116.9 \pm 24.2$ & $115.1 \pm 21.4$ & 0.394 \\
\hline P amp I (mV) & $0.08 \pm 0.03$ & $0.09 \pm 0.03$ & 0.001 \\
\hline \multicolumn{4}{|l|}{ Echo parameters } \\
\hline LA size (mm) & $42.8 \pm 6.4$ & $41.1 \pm 6.2$ & 0.002 \\
\hline $\operatorname{LVEF}(\%)$ & $62.9 \pm 8.1$ & $63.9 \pm 7.8$ & 0.158 \\
\hline $\mathrm{E} / \mathrm{E}^{\prime}$ & $10.2 \pm 3.8$ & $10.1 \pm 4.2$ & 0.824 \\
\hline \multicolumn{4}{|l|}{ LA volume on CT (mL) } \\
\hline LA & $72.1 \pm 21.5$ & $70.0 \pm 22.3$ & 0.329 \\
\hline Venous LA & $22.6 \pm 8.4$ & $22.3 \pm 8.4$ & 0.714 \\
\hline LA appendage & $5.9 \pm 2.7$ & $5.7 \pm 2.9$ & 0.480 \\
\hline Anterior LA & $42.8 \pm 14.9$ & $41.3 \pm 15.4$ & 0.309 \\
\hline \multicolumn{4}{|l|}{ LA voltage (mV) } \\
\hline Mean LA & $1.07 \pm 0.51$ & $1.20 \pm 0.62$ & 0.056 \\
\hline Venous LA & $0.89 \pm 0.67$ & $1.00 \pm 0.79$ & 0.219 \\
\hline LA appendage & $2.11 \pm 1.25$ & $2.40 \pm 1.47$ & 0.070 \\
\hline \multicolumn{4}{|c|}{ Anterior LA Conduction velocity (m/sec) } \\
\hline Anterior & $0.55 \pm 0.25$ & $0.57 \pm 0.30$ & 0.727 \\
\hline Posterior & $0.60 \pm 0.31$ & $0.65 \pm 0.37$ & 0.248 \\
\hline \multicolumn{4}{|l|}{ Procedure } \\
\hline Procedure time (min) & $207.0 \pm 61.6$ & $185.2 \pm 37.8$ & $<0.001$ \\
\hline Fluoroscopic time (min) & $52.5 \pm 21.7$ & $45.7 \pm 27.1$ & 0.003 \\
\hline
\end{tabular}


Ablation time (min)

Genotype distribution*

rs2200733 (4q25)

rs6843082 (PITX2)

rs2106261 (ZFHX3)

$94.6 \pm 31.1$

$83.9 \pm 24.4$

$<0.001$

F, atrial fibrillation; BMI, body mass index; CHF, congestive heart failure; CT, computed tomography; DM, diabetes mellitus; ECG, electrocardiogram; LA, left atrium; LVEF, left ventricular ejection fraction; PAF, paroxysmal atrial fibrillation; TIA, transient ischemic attack.

*Percentage of risk allele carriers in dominant model

rs 13376333 of KCNN3 was not associated with AF.9 These differences could be explained by intrinsic racial differences in myocardial membrane stability, myocardial conduction pathways, or genetic polymorphisms leading to different susceptibility to the development of AF. Another possibility is that the difference could be a result of complicated gene-gene and geneenvironment interactions, which can dilute or accentuate genetic effects in complex traits such as AF.

\section{Potential mechanism of genetic polymorphisms for the development of $\mathrm{AF}$}

Clinical studies have demonstrated that myocardial sleeves in PVs generate ectopic beats that play a substantial role in initiating and maintaining AF. ${ }^{14}$ Electrical isolation of those sleeves is a cornerstone of most AF ablation procedures. PITX2c-deficient mice do not develop pulmonary myocardial sleeves. ${ }^{6}$ Expression of PITX2 is remodeled during cardiac looping, becoming localized to the ventral portion of the developing ventricular chambers, while maintaining distinct left-sided atrial expression. ${ }^{20}$ In addition, PITX 2 might also trigger AF by altering the conduction properties of the cardiac conduction system. Wang et al. ${ }^{21}$ have demonstrated that PITX2 haplo insufficiency predisposes individuals AF in electrically stimulated adult mice. Notably, $A N K 2$, a gene known to play essential roles in in the localization and membrane stabilization of ion transporters and ion channels in disorders such as familial long QT syndrome, sinus node dysfunction, and AF, is also located near rs $2200733 .^{22}$ The ZFHX3 gene on chromosome 16q22 encodes a transcription factor that was originally identified as a regulator of alpha-fetoprotein expression. ${ }^{23} \mathrm{ZFHX} 3$ has been associated with Kawasaki disease, as well as malignancies such as prostate cancer. ${ }^{24}$
However, in human cardiac and pulmonary tissue, its expression pattern and mechanism are not fully known. KCNN channels are also expressed in vascular endothelial cells, and suppression of KCNN3 expression in a mouse model has been associated with increased blood pressure. ${ }^{25}$ In a rabbit burst-pacing model designed to simulate pulmonary venous ectopy, pharmacologic blockade of KCNN channels inhibited pacing-induced shortening of pulmonary venous and atrial action potential duration. ${ }^{26}$ Shortening of the atrial action potential duration reduces the refractory period of atrial myocytes and promotes re-entry, an important mechanism for the development and maintenance of AF. Although the association of rs13376333 in KCNN3 with AF was not demonstrated in the present study, future research will be warranted to further investigate the association between them and elucidate the functional pathways.

\section{The genetic polymorphisms and phenotypes of AF}

Very few studies have evaluated the correlation of genetic variants, $\mathrm{AF}$ phenotypes, and response to $\mathrm{AF}$ therapies. Firouzi et al. ${ }^{27}$ demonstrated increased dispersion of atrial refractoriness in patients with structurally normal hearts who carried the AA genotype of the connexin 40-promoter polymorphism. Husser et al. ${ }^{28}$ found lower AF rates on surface ECG in individuals with the KCNE1 GG genotype. Here, we found the association of genetic polymorphisms with phenotypes of AF patients. Increased risk of AF from 1.04 to 3.9-fold has been observed in patients with prolonged PR interval, and prolonged PR interval is consistently one of the most significant phenotypes related to $\mathrm{AF} .{ }^{10} \mathrm{PR}$ interval is a measure of atrial and atrio-ventricular nodal conduction, and can reflect left or right atrial hypertrophy or enlargement. A recent genome-wide association study of PR interval found links 

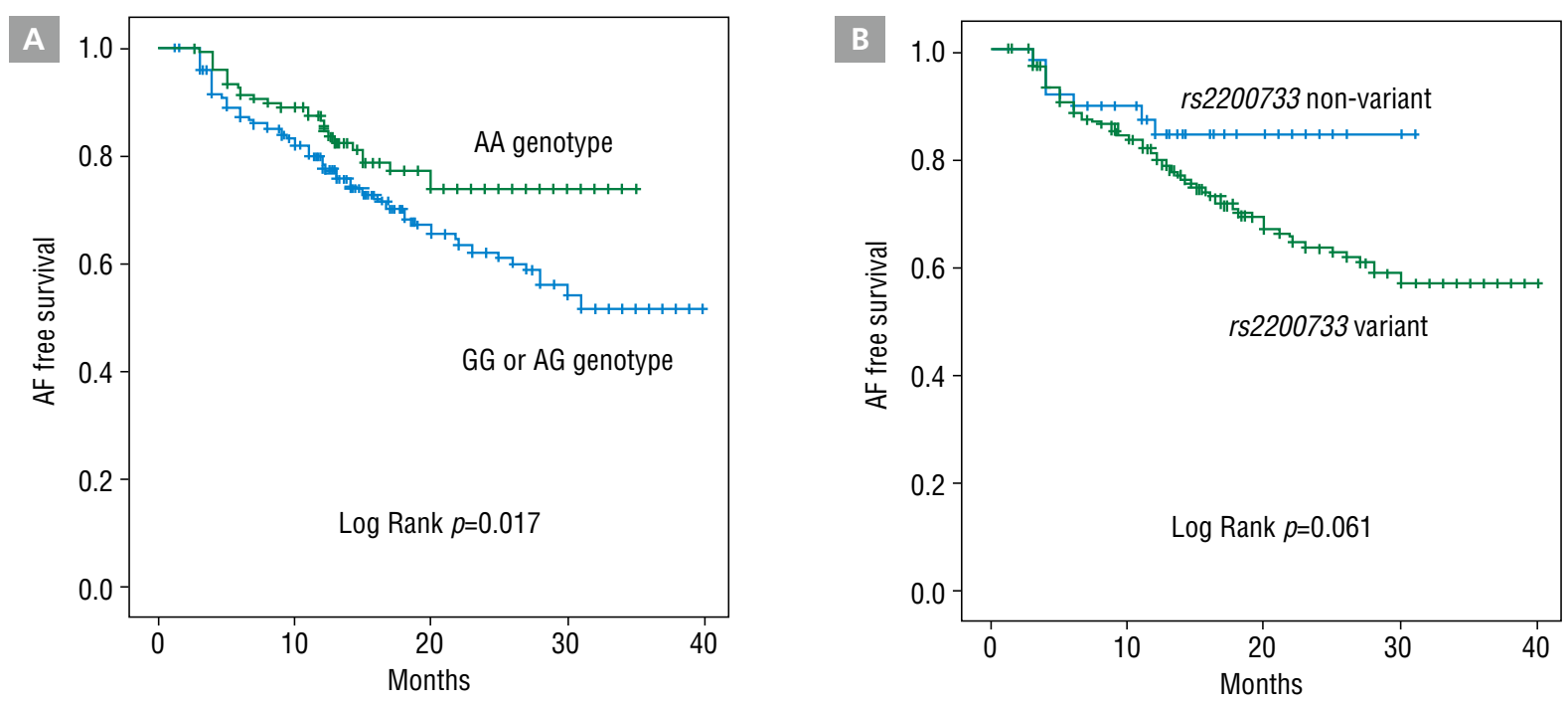

Figure 3. (A) Kaplan-Meier curves of AF free survival in patients with and without the rs2200733 variant allele after RFCA of AF. (B) Kaplan-Meier curves of AF free survival in patients with and without the rs2106261 variant allele after RFCA of AF. $\mathrm{AF}$, atrial fibrillation; RFCA, radiofrequency catheter ablation.



Figure 4. Kaplan-Meier curves of AF free survival according to the number of variant alleles of rs2200733 and rs2106261 (Group A: no variant; $n=15$, Group B: 1 variant; $n=158$, Group C: 2 variants; $n=439$ ) $\mathrm{AF}$, atrial fibrillation.

between PR interval and AF related genes such as CAV1-CAV2, NKX2-5, SOX5, SCN10A, SCN5A, and numerous other genes. ${ }^{29}$ Our AF cohort shows a significant increase in PR interval and negative $\mathrm{P}$ wave terminal force in Lead $\mathrm{V}_{1}$ among patients with the rs2200733 risk allele. Negative $\mathrm{P}$ wave terminal force in Lead $V_{1}$ is indicative of LA conduction impairment, or hypertrophy. Our results indicate that LA hypertrophy, or enlargement modulated by the $4 \mathrm{q} 25$ genetic variant, may increase the risk of AF development. Catheter ablation is one of the most effective treatments for patients with drug-refractory PAF or PeAF..$^{14}$ Many positive outcomes have emerged from the therapy, but adverse effects resulting from scar tissue formation and inability to restore the function of the treated myocardium, as well as complications related to the procedure remain as concerns. Therefore, a better way to predict recurrence of AF after catheter ablation is required. The exact mechanism of AF recurrence after catheter ablation remains elusive. However, multiple studies have shown an impaired clinical response to RFCA of AF in patients carrying the 4q25 rs2200733 variant allele. In a study by Shoemaker ${ }^{30}$, a cohort of 378 patients who underwent catheterbased AF ablations showed 24\% shorter recurrence-free time in patients with the rs 2200733 risk allele compared to those that did not carry the risk allele. In our study, even though the association of rs2200733 with the recurrence of AF after RFCA was not statistically significant, a graded risk of AF recurrence was observed with increasing number of risk alleles at the $4 \mathrm{q} 25$ and ZFHX3 loci. The combination of rs2200733 and rs2106261 might serve as a risk factor for increased AF recurrence after catheter ablation. Our findings suggest that multi-marker allele 
Table 6. Cox regression analysis of AF recurrence after catheter ablation

\begin{tabular}{lccc}
\hline & Hazard Ratio & $95 \% \mathrm{Cl}$ & $P$ value \\
\hline Univariate analysis & & & 0.072 \\
Age & 1.018 & $0.998-1.037$ & 0.283 \\
Male gender & 1.334 & $0.788-2.257$ & $<0.001$ \\
PeAF & 2.181 & $1.449-3.283$ & 0.464 \\
HTN & 0.857 & $0.568-1.294$ & 0.416 \\
DM & 1.266 & $0.717-2.236$ & 0.637 \\
CHADS 2 & 1.048 & $0.864-1.271$ & 0.001 \\
LA size & 1.057 & $1.023-1.091$ & 0.006 \\
Number of variant & 1.536 & $1.133-2.084$ & \\
\hline Multivariate analysis & & & 0.004 \\
PeAF & 1.677 & $1.176-2.381$ & 0.105 \\
LA size & 1.023 & $0.995-1.051$ & 0.015 \\
Number of variants & 1.552 & $1.099-2.222$ & \\
\hline
\end{tabular}

AF, atrial fibrillation; $\mathrm{Cl}$, confidence interval; DM, diabetes mellitus; HTN, hypertension; LA, left atrium; PeAF, persistent AF.

combinations can be used as a clinical tool for the selection of patients for AF ablation. Further research will be warranted to determine whether risk stratification and patient management can be improved by the incorporation of genetic findings.

\section{Study limitations}

We analyzed the SNPs in a Korean population undergoing catheter ablation. Therefore, our findings cannot be generalized to all types of AF. When applying SNPs as predictors and targets of therapy for AF, possible racial differences in the association between SNPs and AF should be considered.

\section{Conclusion}

This study shows the common AF susceptibility SNP alleles of $4 \mathrm{q} 25$ and $Z F H X 3$, are associated with the electroanatomical characteristics of LA and the clinical outcomes of catheter ablation in Korean patients with AF. These findings imply that gene variants may have a potential role for risk stratification of ablation therapy and post-ablation management.

\section{References}

1) Fuster V, Ryden LE, Cannom DS, Crijns HJ, Curtis AB, Ellenbogen KA, Halperin JL, Le Heuzey JY, Kay GN, Lowe JE, Olsson SB, Prystowsky EN, Tamargo JL, Wann S, Smith SC, Jr., Jacobs AK, Adams CD, Anderson JL, Antman EM, Hunt SA, Nishimura R, Ornato JP, Page RL, Riegel B, Priori SG, Blanc JJ, Budaj A, Camm AJ, Dean V, Deckers JW, Despres C, Dickstein K, Lekakis J, McGregor K, Metra M, Morais J, Osterspey A and Zamorano JL. ACC/AHA/ESC 2006 Guidelines for the Management of Patients with Atrial Fibrillation: a report of the American College of Cardiology/American Heart Association Task Force on Practice Guidelines and the European Society of Cardiology Committee for Practice Guidelines (Writing Committee to Revise the 2001 Guidelines for the Management of Patients With Atrial Fibrillation): developed in collaboration with the European Heart Rhythm Association and the Heart Rhythm Society. Circulation. 2006;114:e257-e354. 
2) Magnani JW, Rienstra M, Lin H, Sinner MF, Lubitz SA, McManus DD, Dupuis J, Ellinor PT and Benjamin EJ. Atrial fibrillation: current knowledge and future directions in epidemiology and genomics. Circulation. 2011;124:1982-1993.

3) Sinner MF, Ellinor PT, Meitinger T, Benjamin EJ and Kaab S. Genome-wide association studies of atrial fibrillation: past, present, and future. Cardiovasc Res. 2011;89:701-709.

4) Kaab S, Darbar D, van Noord C, Dupuis J, Pfeufer A, NewtonCheh C, Schnabel R, Makino S, Sinner MF, Kannankeril PJ, Beckmann BM, Choudry S, Donahue BS, Heeringa J, Perz S, Lunetta KL, Larson MG, Levy D, MacRae CA, Ruskin JN, Wacker A, Schomig A, Wichmann HE, Steinbeck G, Meitinger T, Uitterlinden AG, Witteman JC, Roden DM, Benjamin EJ and Ellinor PT. Large scale replication and meta-analysis of variants on chromosome 4q25 associated with atrial fibrillation. Eur Heart J. 2009;30:813-819.

5) Gudbjartsson DF, Holm H, Gretarsdottir S, Thorleifsson G, Walters GB, Thorgeirsson G, Gulcher J, Mathiesen EB, Njolstad I, Nyrnes A, Wilsgaard T, Hald EM, Hveem K, Stoltenberg C, Kucera G, Stubblefield T, Carter S, Roden D, Ng MC, Baum L, So WY, Wong KS, Chan JC, Gieger C, Wichmann HE, Gschwendtner A, Dichgans M, Kuhlenbaumer G, Berger K, Ringelstein EB, Bevan S, Markus HS, Kostulas K, Hillert J, Sveinbjornsdottir S, Valdimarsson EM, Lochen ML, Ma RC, Darbar D, Kong A, Arnar DO, Thorsteinsdottir U and Stefansson K. A sequence variant in $Z F H X 3$ on 16q22 associates with atrial fibrillation and ischemic stroke. Nat Genet. 2009;41:876-878.

6) Mommersteeg MT, Brown NA, Prall OW, de Gier-de Vries C, Harvey RP, Moorman AF and Christoffels VM. Pitx2c and Nkx2-5 are required for the formation and identity of the pulmonary myocardium. Circ Res. 2007;101:902-909.

7) Gudbjartsson DF, Arnar DO, Helgadottir A, Gretarsdottir S, Holm H, Sigurdsson A, Jonasdottir A, Baker A, Thorleifsson G, Kristjansson K, Palsson A, Blondal T, Sulem P, Backman VM, Hardarson GA, Palsdottir E, Helgason A, Sigurjonsdottir R, Sverrisson JT, Kostulas K, Ng MC, Baum L, So WY, Wong KS, Chan JC, Furie KL, Greenberg SM, Sale M, Kelly P, MacRae CA, Smith EE, Rosand J, Hillert J, Ma RC, Ellinor PT, Thorgeirsson G, Gulcher JR, Kong A, Thorsteinsdottir U and Stefansson K. Variants conferring risk of atrial fibrillation on chromosome $4 \mathrm{q} 25$. Nature. 2007;448:353-357.

8) Shi L, Li C, Wang C, Xia Y, Wu G, Wang F, Xu C, Wang P, Li X, Wang D, Xiong X, Bai Y, Liu M, Liu J, Ren X, Gao L, Wang B,
Zeng Q, Yang B, Ma X, Yang Y, Tu X and Wang QK. Assessment of association of rs2200733 on chromosome 4q25 with atrial fibrillation and ischemic stroke in a Chinese Han population. Hum Genet. 2009; 126:843-849.

9) Ellinor PT, Lunetta KL, Glazer NL, Pfeufer A, Alonso A, Chung MK, Sinner MF, de Bakker PI, Mueller M, Lubitz SA, Fox E, Darbar D, Smith NL, Smith JD, Schnabel RB, Soliman EZ, Rice KM, Van Wagoner DR, Beckmann BM, van Noord C, Wang K, Ehret GB, Rotter JI, Hazen SL, Steinbeck G, Smith AV, Launer LJ, Harris TB, Makino S, Nelis M, Milan DJ, Perz S, Esko T, Kottgen A, Moebus S, Newton-Cheh C, Li M, Mohlenkamp S, Wang TJ, Kao WH, Vasan RS, Nothen MM, MacRae CA, Stricker BH, Hofman A, Uitterlinden AG, Levy D, Boerwinkle E, Metspalu A, Topol EJ, Chakravarti A, Gudnason V, Psaty BM, Roden DM, Meitinger T, Wichmann HE, Witteman JC, Barnard J, Arking DE, Benjamin EJ, Heckbert SR and Kaab S. Common variants in KCNN3 are associated with lone atrial fibrillation. Nat Genet. 2010;42:240-244.

10) Cheng S, Keyes MJ, Larson MG, McCabe EL, Newton-Cheh C, Levy D, Benjamin EJ, Vasan RS and Wang TJ. Long-term outcomes in individuals with prolonged PR interval or first-degree atrioventricular block. JAMA. 2009;301:2571-2577.

11) Darbar D, Jahangir A, Hammill SC and Gersh BJ. P wave signalaveraged electrocardiography to identify risk for atrial fibrillation. Pacing Clin Electrophysiol. 2002;25:1447-1453.

12) Zaman AG, Archbold RA, Helft G, Paul EA, Curzen NP and Mills PG. Atrial fibrillation after coronary artery bypass surgery: a model for preoperative risk stratification. Circulation. 2000;101:14031408.

13) Rienstra M, McManus DD and Benjamin EJ. Novel risk factors for atrial fibrillation: useful for risk prediction and clinical decision making? Circulation. 2012;125:e941-e946.

14) Calkins H, Kuck KH, Cappato R, Brugada J, Camm AJ, Chen SA, Crijns HJ, Damiano RJ, Jr., Davies DW, DiMarco J, Edgerton J, Ellenbogen K, Ezekowitz MD, Haines DE, Haissaguerre M, Hindricks G, Iesaka Y, Jackman W, Jalife J, Jais P, Kalman J, Keane D, Kim YH, Kirchhof P, Klein G, Kottkamp H, Kumagai K, Lindsay BD, Mansour M, Marchlinski FE, McCarthy PM, Mont JL, Morady F, Nademanee K, Nakagawa H, Natale A, Nattel S, Packer DL, Pappone C, Prystowsky E, Raviele A, Reddy V, Ruskin JN, Shemin RJ, Tsao HM, Wilber D, Heart Rhythm Society Task Force on C and Surgical Ablation of Atrial F. 2012 HRS/EHRA/ ECAS expert consensus statement on catheter and surgical 
ablation of atrial fibrillation: recommendations for patient selection, procedural techniques, patient management and followup, definitions, endpoints, and research trial design: a report of the Heart Rhythm Society (HRS) Task Force on Catheter and Surgical Ablation of Atrial Fibrillation. Developed in partnership with the European Heart Rhythm Association (EHRA), a registered branch of the European Society of Cardiology (ESC) and the European Cardiac Arrhythmia Society (ECAS); and in collaboration with the American College of Cardiology (ACC), American Heart Association (AHA), the Asia Pacific Heart Rhythm Society (APHRS), and the Society of Thoracic Surgeons (STS). Endorsed by the governing bodies of the American College of Cardiology Foundation, the American Heart Association, the European Cardiac Arrhythmia Society, the European Heart Rhythm Association, the Society of Thoracic Surgeons, the Asia Pacific Heart Rhythm Society, and the Heart Rhythm Society. Heart Rhythm. 2012;9:632-696.e21.

15) Husser D, Adams V, Piorkowski C, Hindricks G and Bollmann A. Chromosome $4 \mathrm{q} 25$ variants and atrial fibrillation recurrence after catheter ablation. J Am Coll Cardiol. 2010;55:747-753.

16) Fox CS, Parise H, D'Agostino RB, Sr., Lloyd-Jones DM, Vasan RS, Wang TJ, Levy D, Wolf PA and Benjamin EJ. Parental atrial fibrillation as a risk factor for atrial fibrillation in offspring. JAMA. 2004;291:2851-2855.

17) Lubitz SA, Yin X, Fontes JD, Magnani JW, Rienstra M, Pai M, Villalon ML, Vasan RS, Pencina MJ, Levy D, Larson MG, Ellinor PT and Benjamin EJ. Association between familial atrial fibrillation and risk of new-onset atrial fibrillation. JAMA. 2010;304:2263-2269.

18) Postma AV, Christoffels VM and Bezzina CR. Developmental aspects of cardiac arrhythmogenesis. Cardiovasc Res. 2011;91:243251.

19) Pennacchio LA, Ahituv N, Moses AM, Prabhakar S, Nobrega MA, Shoukry M, Minovitsky S, Dubchak I, Holt A, Lewis KD, Plajzer-Frick I, Akiyama J, De Val S, Afzal V, Black BL, Couronne $\mathrm{O}$, Eisen MB, Visel A and Rubin EM. In vivo enhancer analysis of human conserved non-coding sequences. Nature. 2006;444:499502.

20) Campione M, Ros MA, Icardo JM, Piedra E, Christoffels VM, Schweickert A, Blum M, Franco D and Moorman AF. Pitx2 expression defines a left cardiac lineage of cells: evidence for atrial and ventricular molecular isomerism in the iv/iv mice. Dev Biol. 2001;231:252-264.
21) Wang J, Klysik E, Sood S, Johnson RL, Wehrens XH and Martin JF. Pitx 2 prevents susceptibility to atrial arrhythmias by inhibiting left-sided pacemaker specification. Proc Natl Acad Sci U S A. 2010;107:9753-9758.

22) Mohler PJ, Schott JJ, Gramolini AO, Dilly KW, Guatimosim S, duBell WH, Song LS, Haurogne K, Kyndt F, Ali ME, Rogers TB, Lederer WJ, Escande D, Le Marec H and Bennett V. Ankyrin-B mutation causes type 4 long-QT cardiac arrhythmia and sudden cardiac death. Nature. 2003;421:634-639.

23) Burgner D, Davila S, Breunis WB, Ng SB, Li Y, Bonnard C, Ling L, Wright VJ, Thalamuthu A, Odam M, Shimizu C, Burns JC, Levin M, Kuijpers TW, Hibberd ML and International Kawasaki Disease Genetics C. A genome-wide association study identifies novel and functionally related susceptibility Loci for Kawasaki disease. PLoS Genet. 2009;5:e1000319.

24) Sun X, Frierson HF, Chen C, Li C, Ran Q, Otto KB, Cantarel BL, Vessella RL, Gao AC, Petros J, Miura Y, Simons JW and Dong JT. Frequent somatic mutations of the transcription factor ATBF1 in human prostate cancer. Nat Genet. 2005;37:407-412.

25) Taylor MS, Bonev AD, Gross TP, Eckman DM, Brayden JE, Bond CT, Adelman JP and Nelson MT. Altered expression of smallconductance Ca2+-activated $\mathrm{K}+$ (SK3) channels modulates arterial tone and blood pressure. Circ Res. 2003;93:124-131.

26) Ozgen N, Dun W, Sosunov EA, Anyukhovsky EP, Hirose M, Duffy HS, Boyden PA and Rosen MR. Early electrical remodeling in rabbit pulmonary vein results from trafficking of intracellular SK2 channels to membrane sites. Cardiovasc Res. 2007;75:758769.

27) Firouzi M, Ramanna $H$, Kok B, Jongsma HJ, Koeleman BP, Doevendans PA, Groenewegen WA and Hauer RN. Association of human connexin 40 gene polymorphisms with atrial vulnerability as a risk factor for idiopathic atrial fibrillation. Circ Res. 2004;95:e29-33.

28) Husser D, Stridh M, Sornmo L, Roden DM, Darbar D and Bollmann A. A genotype-dependent intermediate ECG phenotype in patients with persistent lone atrial fibrillation genotype ECG-phenotype correlation in atrial fibrillation. Circ Arrhythm Electrophysiol. 2009;2:24-28.

29) Pfeufer A, van Noord C, Marciante KD, Arking DE, Larson MG, Smith AV, Tarasov KV, Muller M, Sotoodehnia N, Sinner MF, Verwoert GC, Li M, Kao WH, Kottgen A, Coresh J, Bis JC, Psaty BM, Rice K, Rotter JI, Rivadeneira F, Hofman A, Kors JA, Stricker 
BH, Uitterlinden AG, van Duijn CM, Beckmann BM, Sauter W, Gieger C, Lubitz SA, Newton-Cheh C, Wang TJ, Magnani JW, Schnabel RB, Chung MK, Barnard J, Smith JD, Van Wagoner DR, Vasan RS, Aspelund T, Eiriksdottir G, Harris TB, Launer LJ, Najjar SS, Lakatta E, Schlessinger D, Uda M, Abecasis GR, MullerMyhsok B, Ehret GB, Boerwinkle E, Chakravarti A, Soliman EZ, Lunetta KL, Perz S, Wichmann HE, Meitinger T, Levy D, Gudnason V, Ellinor PT, Sanna S, Kaab S, Witteman JC, Alonso
A, Benjamin EJ and Heckbert SR. Genome-wide association study of PR interval. Nat Genet. 2010;42:153-159.

30) Benjamin Shoemaker M, Muhammad R, Parvez B, White BW, Streur M, Song Y, Stubblefield T, Kucera G, Blair M, Rytlewski J, Parvathaneni S, Nagarakanti R, Saavedra P, Ellis CR, Patrick Whalen S, Roden DM and Darbar RD. Common atrial fibrillation risk alleles at $4 \mathrm{q} 25$ predict recurrence after catheterbased atrial fibrillation ablation. Heart Rhythm. 2013;10:394-400. 